XIV Simpósio Nacional de Sistemas Prediais

Gestão, Eficiência e Sustentabilidade

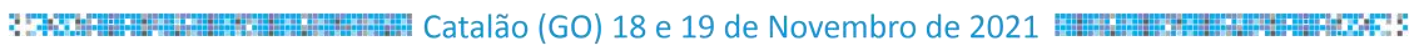

\author{
REALIDADE AUMENTADA EM SISTEMAS HIDROSSANITÁRIOS RESIDENCIAIS \\ Augmented Reality In Residential Plumbing Systems \\ ENDRES, João Pedro1; BRIÃO, Vandré Barbosa²; FERNANDES, Vera Maria Cartana³ \\ Recebido em 15 de julho de 2021, aprovado em 28 de outubro de 2021, publicado em 18 de novembro de 2021
}

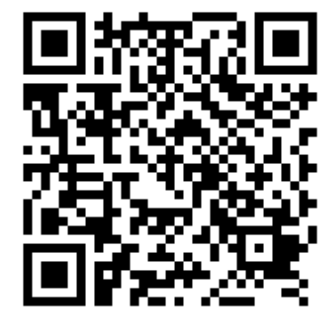

Palavras-chave:

Construção,

Inovação,

Tecnologia da

informação e

comunicação.

Keywords:

Construction,

Innovation,

Information and communication technology.
RESUMO: O uso de RA nos projetos dos sistemas hidrossanitários teve como objetivo estudar a aplicabilidade da Realidade Aumentada (RA) na execução dos projetos de sistemas hidrossanitários em edificações. A metodologia da pesquisa seguiu primeiramente com testes de precisão do método de rastreamento por visualização de marcadores e do aplicativo Augin, constatando nos piores casos $0,5 \mathrm{~cm}$ de distorção do sistema virtual para o real. Depois disso foram aplicados modelos em RA em 6 edificações de diferentes padrões construtivos. Por fim se fez uma pesquisa de satisfação com os instaladores de cada edificação e constatou-se excelente aceitação do método, não havendo diferenças nos resultados em relação ao padrão de qualidade das obras, universalizando assim o uso dessa tecnologia no setor da construção civil.

\begin{abstract}
The use of AR in the plumbling systems design aimed to study the applicability of Augmented Reality (AR) in the execution of plumbling system projects in buildings. The research methodology followed, firstly, with accuracy tests of the marker visualization tracking method and the Augin application, noting in the worst cases $0.5 \mathrm{~cm}$ of distortion from the virtual system to the real one. Afterwards, models in RA were applied in 6 buildings of different construction standards. Finally, a satisfaction survey was carried out with the installers of each building and found an excellent acceptance of the method, with no differences in the results in relation to the quality standard, thus universalizing the use of this technology in the civil construction sector.
\end{abstract}

\title{
CONTATO DOS AUTORES:
}

${ }^{1}$ ENDRES, João Pedro: Eng. Civil MSc. Universidade de Passo Fundo, joaopendres94@gmail.com

2 BRIÃO, Vandré Barbosa: Eng. De Alimentos, Dr. Professor da Universidade de Passo Fundo, vandre@upf

${ }^{3}$ FERNANDES, Vera Maria Cartana: Enga. Civil Dra. Professora da Universidade de Passo Fundo, cartana@upf.br 


\section{INTRODUÇÃO}

A maneira como se visualiza os projetos de sistemas hidrossanitários é de extrema importância visto as inúmeras possibilidades de interferências. Os projetistas da área, em conjunto com profissionais da computação, têm estudado o desenvolvimento de novas tecnologias de visualização dos projetos hidrossanitários que tendem a facilitar o gerenciamento de suas informações. Essa nova forma de visualização inclui a realidade aumentada (RA).

Levando em consideração as dificuldades encontradas nas interpretações dos projetos de sistemas hidrossanitários e o atual cenário da realidade aumentada como possível solução para essas dificuldades, justifica-se a importância do desenvolvimento da presente pesquisa. A mesma buscou ajudar os instaladores no melhor entendimento dos projetos de sistemas hidrossanitários. Uma pesquisa realizada por Mesquita et al. (2018) demonstrou que a tridimensionalidade dos elementos com a utilização de RA facilitou o entendimento das áreas de projeto até mesmo para leigos. Pode o cenário atual ser favorável para o desenvolvimento e avanço da execução dos projetos de sistemas hidrossanitários?

Dessa forma, o objetivo geral da pesquisa é estudar a aplicabilidade da Realidade Aumentada (RA) na execução dos projetos de sistemas hidrossanitários em edificações. Para alcançar este objetivo foram contemplados os seguintes objetivos específicos:

A) Aplicar os conceitos de RA na execução dos projetos de sistemas hidrossanitários;

B) Avaliar possíveis desvios entre o modelo de realidade aumentada e os sistemas hidrossanitários;

C) Realizar uma pesquisa de satisfação quanto ao uso de RA em projetos hidrossanitários, verificando a aceitação dos instaladores.

\section{REVISÃO BIBLIOGRÁFICA}

\subsection{Realidade aumentada}

A realidade aumentada (RA) é a imagem do mundo real com introdução de elementos virtuais (EL AMMARI e HAMMAD, 2019). Esta ferramenta faz com que objetos virtuais e objetos reais coexistam no mesmo espaço do mundo real (AZUMA, 2001). A Figura 1 demonstra a aplicação de RA em um manual do proprietário. 


\section{Figura 1 - Exemplo de aplicação de realidade aumentada}

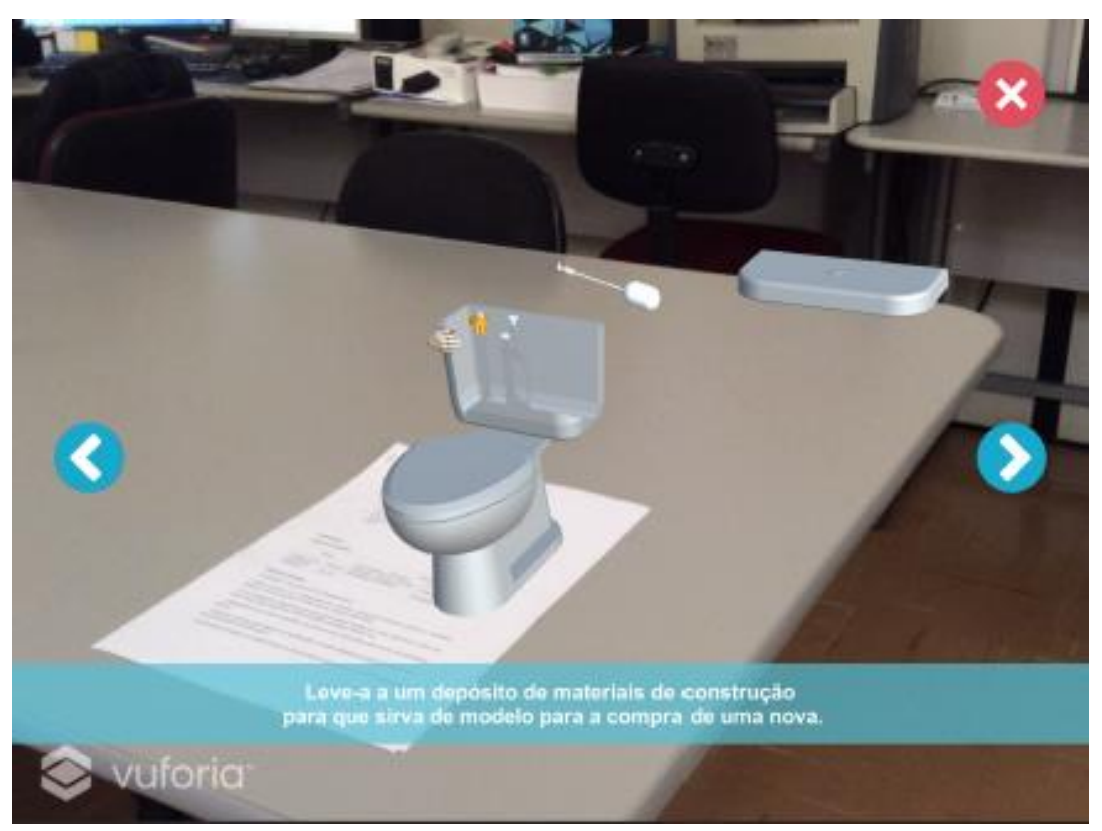

Fonte: Moreira (2018).

\subsection{Realidade aumentada na construção civil}

Uma pesquisa realizada no portal de periódicos da CAPES (www.periodicos.capes.gov.br) retornou 4636 resultados, e cerca de um terço dessas publicações (1535) são dos últimos cinco anos. Isso demonstra que a realidade aumentada tem ganhado espaço nos diferentes ambientes da construção civil e arquitetura.

\section{METODOLOGIA}

A Figura 2 representa a ordem metodológica que adotou-se para a realização da pesquisa.

Figura 2 - Exemplo de aplicação de realidade aumentada

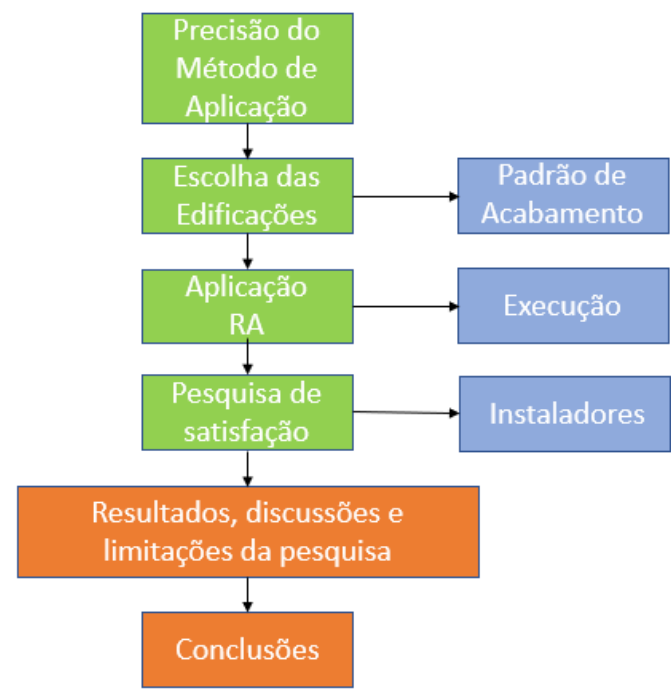

Fonte: Próprio Autor (2021). 


\subsection{Precisão do método de aplicação}

No software QiBuilder lançou-se 3 comprimentos diferentes de tubulação horizontal, sendo estes de 2 metros, 4 metros e 8 metros, isto para avaliar uma possível distorção no alinhamento do sistema em relação a distância. Esses testes foram realizados em uma edificação na cidade de Horizontina-RS. O protocolo seguiu o procedimento:

a) Desenhar tubulação no software QiBuilder;

b) Importar desenho para aplicativo Augin, locando reference tracker virtual;

c) Projetar nível a laser na mesma altura da tubulação virtual;

d) Locar reference tracker ${ }^{1}$ físico;

e) Operar o sistema em RA a partir do uso de um celular;

f) Medir manualmente a distância do eixo da tubulação virtual com a linha laser de referência.

Sobre uma parede lançou-se então o nível a laser na mesma altura de 1,5 metros do lançamento virtual do tubo para assim conseguir medir manualmente a distância aproximada do eixo do tubo com a linha de referência (laser).

\subsection{Edificações}

A presente pesquisa foi realizada em 6 edificações que estavam em processos de construção, na cidade de Horizontina - Rio Grande do Sul. Com isso as construtoras desenvolvedoras dos projetos de sistemas hidrossanitários disponibilizaram os projetos utilizados nas construções. Para a classificação das casas utilizou-se a cartilha elaborada pelo SINDUSCON-MG (2007), sendo trabalhadas nessa pesquisa residências unifamiliares padrão baixo, padrão normal e padrão alto.

\subsection{Aplicação RA}

Os projetos hidrossanitários foram elaborados no software QiBuilder versão 2020 da empresa AltoQl; Já a modelagem em RA foi realizada no aplicativo nacional Augin, com método de rastreamento por visualização a partir de $Q R$ code, com leitura via celulares.

\subsection{Pesquisa de Satisfação}

Ao todo foram entrevistados 6 instaladores, 1 de cada edificação. O questionário foi composto por perguntas objetivas, tendo cada pergunta 5 respostas possíveis que foram pontuadas para computar graficamente os resultados. Essa pontuação foi definida como Horrível (2), Ruim (4), Bom (6), Muito Bom (8) e Excelente (10). A Figura 3 demonstra o questionário com as possíveis respostas.

\footnotetext{
${ }^{1}$ Reference Tracker é um ponto de referência criado no website Augin para ajudar a locar um modelo em RA na escala 1:1.
} 
Figura 3 - Pesquisa de Satisfação

Perguntas para o Instalador:

1) Colaboração da RA durante a execução dos projetos dos sistemas hidrossanitários?
()Excelente ()Muito Bom ()Bom
() Ruim
() Horrivel

2) Ajuda da RA para prever a execução do sistema hidrossanitário, evitando retrabalho?
()Excelente
()Muito Bom ()Bom
() Ruim
() Horrivel

3) Facilidade em manusear o dispositivo móvel para a prática da RA? Escreva logo abaixo qual o dispositivo móvel utilizado (próprio ou da pesquisa)

()Excelente ()Muito Bom ()Bom () Ruim () Horrível

4) Comparando essa tecnologia (RA) de visualização do projeto com o sistema convencional (planta baixa e isométricos, impressos), houveram avanços?
()Excelente ()Muito Bom ()Bom
() Ruim
() Horrível

5) O que achou do método de rastreamento por visualização de marcadores $Q R$ code dos modelos em RA?
()Excelente
()Muito Bom
()Bom
() Ruim
() Horrivel

6) Utilizando RA como permaneceu a execução em relação ao projeto?

()Excelente ()Muito Bom ()Bom () Ruim () Horrível

Fonte: Próprio Autor (2021).

\section{RESULTADOS E DISCUSSÃO}

\subsection{Avaliação da precisão do aplicativo Augin}

Após locar o sistema de referência físico, alinhar o laser na posição da tubulação, se fez a aplicação do aplicativo Augin como pode ser visto na Figura 4.

\section{Figura 4 - Sistema aplicado in loco (1)}

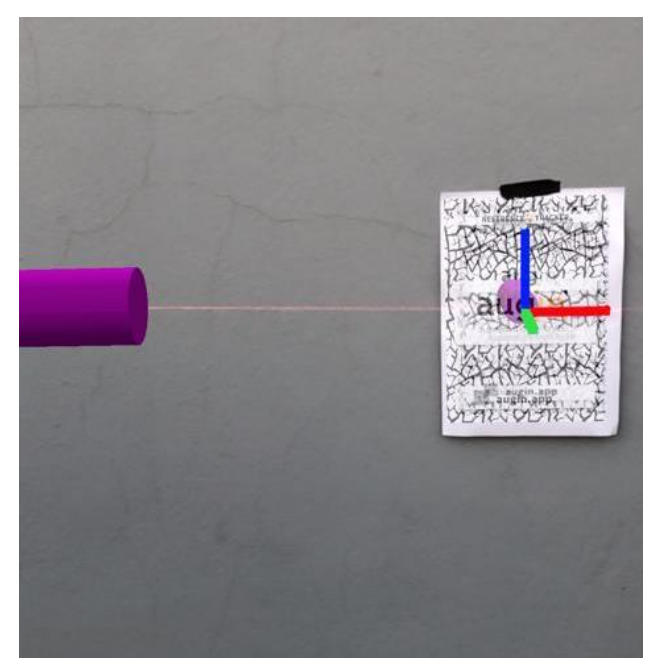

Fonte: Próprio Autor (2021). 
Como pode ser visto na Figura 4 a tubulação está completamente alinhada ao laser, porém o reference tracker está dentro do campo de visão do dispositivo móvel. Ao girar a câmera tirando o ponto de referência do campo de visão notou-se um pequeno desalinhamento de aproximadamente $0,5 \mathrm{~cm}$ como demonstrado na Figura 5.

\section{Figura 5 - Sistema aplicado in loco (2)}

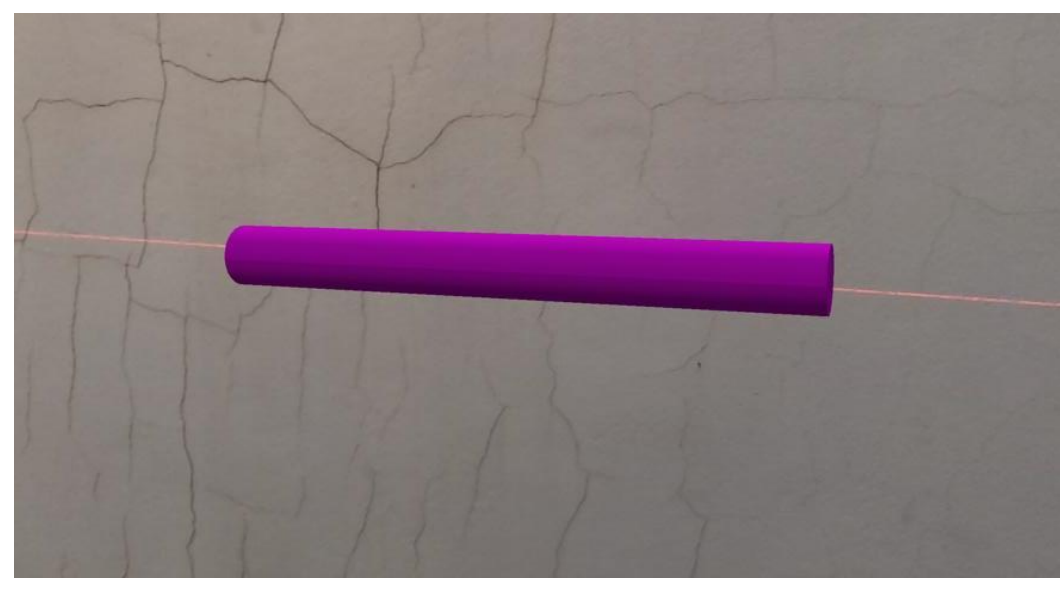

Fonte: Próprio Autor (2021).

Os testes foram feitos três vezes sucessivos para cada comprimento de tubulação e os resultados foram praticamente os mesmos, nunca variando para mais de $0,5 \mathrm{~cm}$. Vale ressaltar que nesses testes apenas a câmera foi rotacionada, permanecendo a pessoa no mesmo local da leitura. Por fim testou-se fazer o deslocamento do dispositivo móvel em relação ao local de leitura, e assim a precisão do sistema foi comprometida ocorrendo o desalinhamento do reference tracker e consequentemente da tubulação, como pode ser visualizado na Figura 6.

\section{Figura 6 - Deslocamento do dispositivo móvel em relação ao local de leitura}

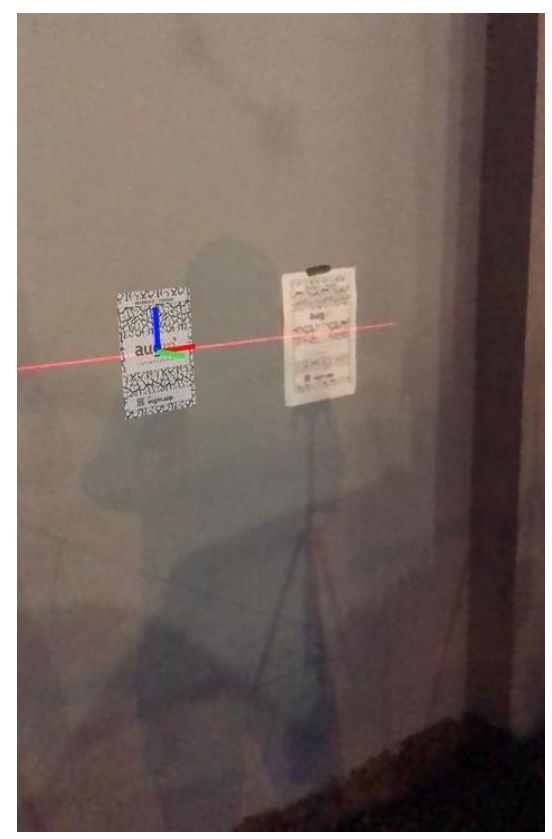

Fonte: Próprio Autor (2021). 
Portanto, a partir destes testes da precisão do sistema constatou-se que o dispositivo móvel deve ficar parado em frente ao reference tracker, podendo ser feito a rotação para os lados para visualização das tubulações, não comprometendo assim a precisão mesmo quando o ponto de referência sai do campo de visão da câmera. Além disso a diferença de $0,5 \mathrm{~cm}$ na precisão do uso do aplicativo Augin pode ser resultado da diferença de casas decimais entre os softwares utilizados, como também pela calibração do giroscópio do dispositivo (SANTOS et al., 2021).

\subsection{Aplicação RA}

A seguir, na Figura 7 apresenta-se a aplicação de RA nas edificações estudadas.

\section{Figura 7 - Aplicação RA}
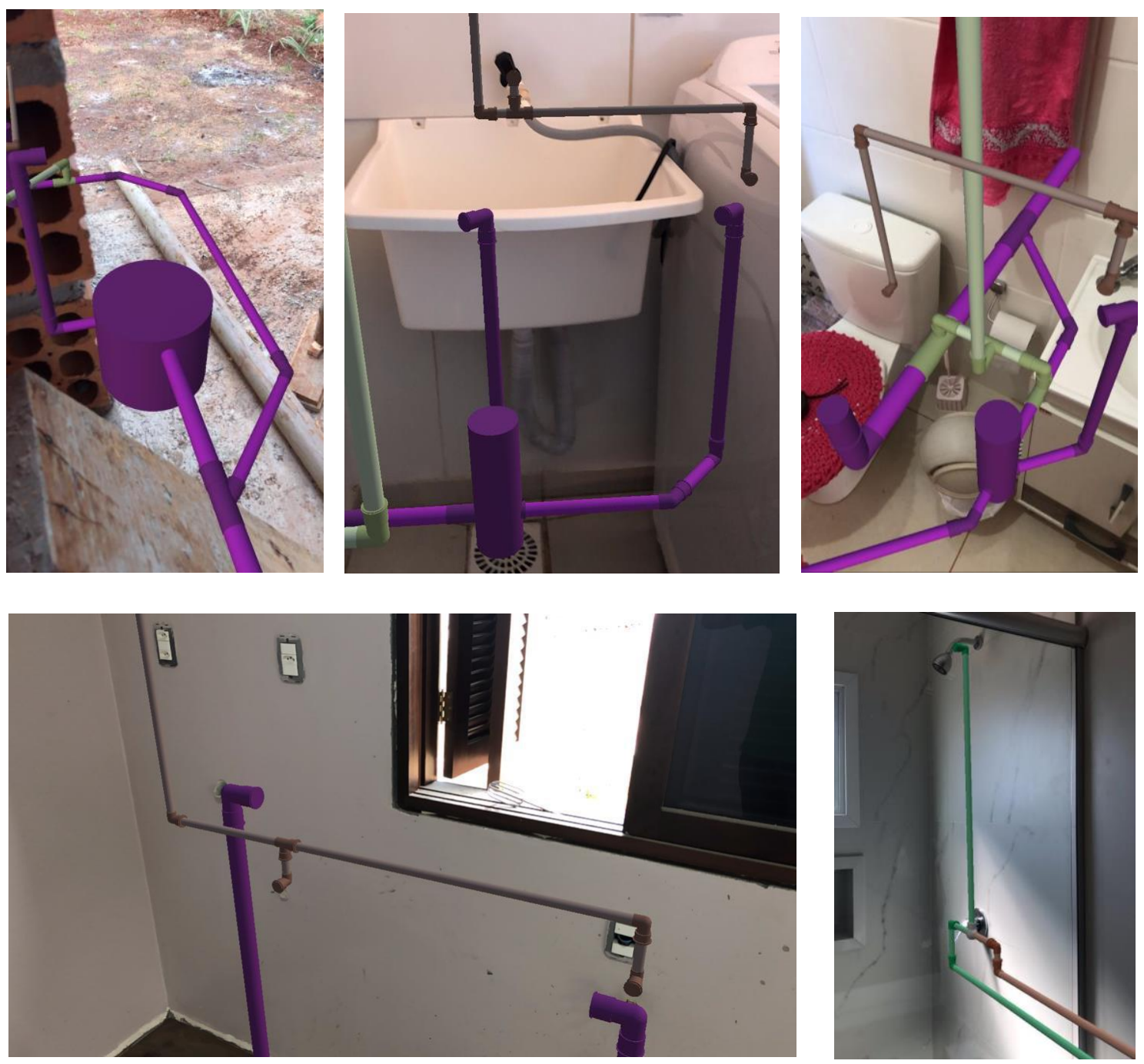

Fonte: Próprio Autor (2021).

\subsection{Pesquisa de satisfação - Instaladores}

O questionário da pesquisa de satisfação com os instaladores foi aplicado em cada uma das edificações (da 1 à 6). As respostas estão registradas no gráfico apresentado pela Figura 8. 
Figura 8 - Pesquisa de Satisfação - Instaladores

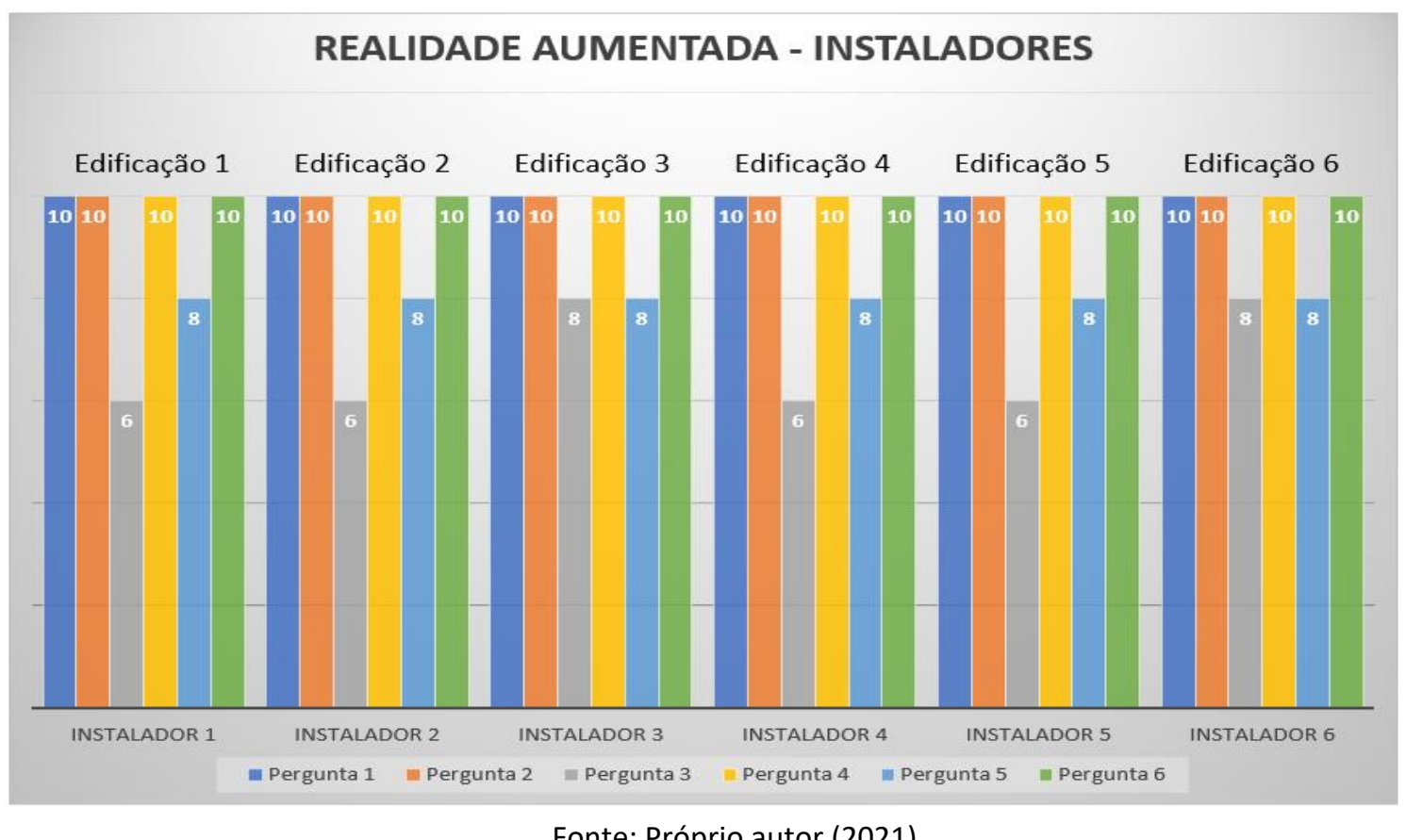

Fonte: Próprio autor (2021).

Para uma melhor conclusão dos resultados analisados com a pesquisa de satisfação gerou-se gráfico com a média e desvio padrão da soma das pontuações de cada entrevistado para cada padrão de edificação, conforme Figura 9. Em relação a classificação das pontuações, a Tabela 1 representa o resultado da pesquisa com os instaladores conforme pontuação determinada para cada tipo de resposta.

Tabela 1 - Resultado da pesquisa com os Instaladores

\begin{tabular}{rlllll}
\hline Classificação & Horrível & Ruim & Bom & Muito bom & Excelente \\
\hline Pontuação & $0-12$ & $13-24$ & $25-36$ & $37-48$ & $49-60$ \\
\hline \multicolumn{6}{c}{ Fonte: Próprio Autor (2021). }
\end{tabular}

Figura 9 - Médias e desvios padrões pesquisa de satisfação Instaladores

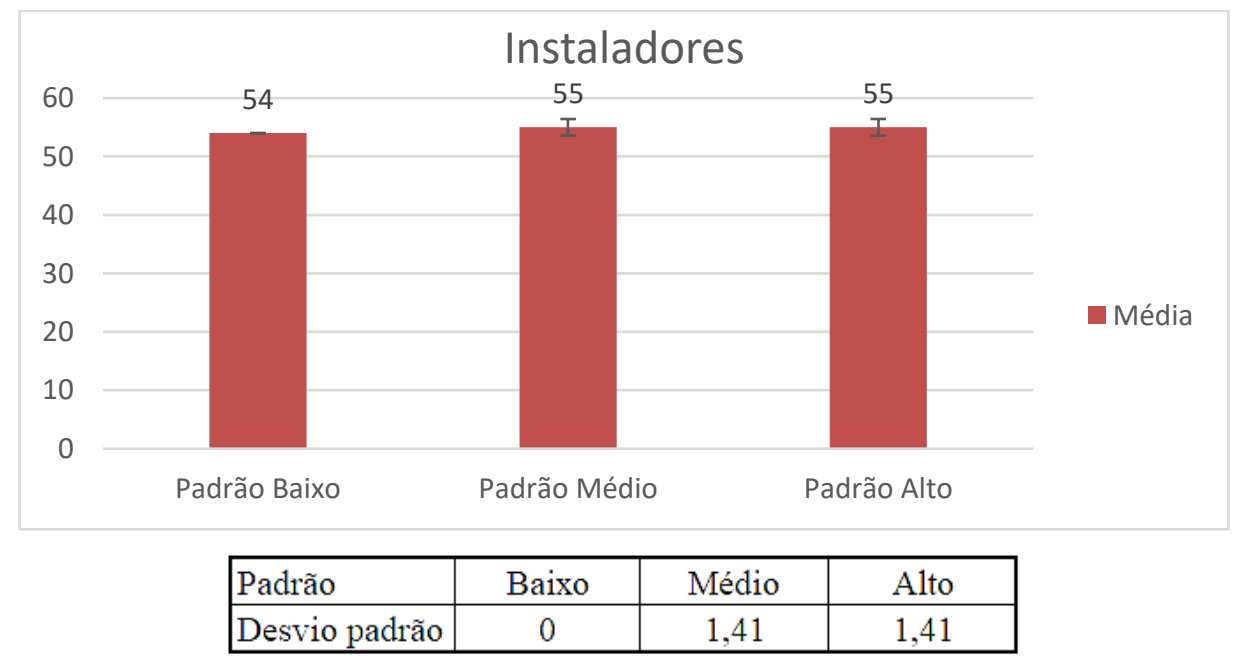

Fonte: Próprio Autor (2021). 
Em relação aos instaladores a pontuação média atingida ficou dentro do intervalo de 48-60, resultando em uma avaliação "excelente". Os padrões das edificações não apresentaram variações significantes de pontuações, o que demonstra uniformidade na aceitação da RA por parte dos instaladores.

Em todas as edificações da presente pesquisa os instaladores optaram pelo uso desta ferramenta tecnológica ao invés do uso do papel (método convencional). Essa mesma preferência também foi constatada na pesquisa de Yeh et al. (2012) onde os operadores receberam as informações de projeto de maneira mais eficiente e precisa, quando comparado ao método convencional, devido ao maior detalhamento dos desenhos. A RA faz com que a carga cognitiva dos profissionais seja reduzida, não precisando mais imaginar as tubulações locadas na obra, o que acaba por liberar recursos mentais fazendo com que os mesmos se concentrem mais na execução do serviço (RE et al., 2016). O uso dessa ferramenta faz com que as pessoas possam visualizar em tempo real o que virá a acontecer (BEHZADI, 2017). Tudo isso faz com que diminua a quantidade de erros de execução, o que acaba por evitar retrabalho e assim desperdício de materiais. Conforme Dallasega et al. (2020) constataram em sua pesquisa, o uso de RA ajuda a melhorar indicadores de desempenho como o tempo de construção, o nível de qualidade como também o nível de sustentabilidade.

Em relação ao uso de dispositivos móveis como smartphones para a aplicação da tecnologia de RA, alguns instaladores que possuíam mais idade, na maioria das vezes apresentaram maiores dificuldades de manuseio. Porém, com o devido treinamento, os mesmos acabavam por aprender e conseguir projetar os sistemas hidrossanitários in loco. Os trabalhadores da construção civil vem preferindo inserir novas tecnologias com interfaces digitais no canteiro de obras e a grande explicação para isso é o ambiente familiar, que também adota novos dispositivos passando, então, muito mais confiança para o aprendizado (YANG et al., 2021). Comparando o modo de visualização em RA com o 3D em tela, o primeiro é muito mais intuitivo o que facilita na aprendizagem da sua utilização (GARBETT et al., 2021). Dessa forma percebe-se que o uso de RA acaba por ser mais vantajoso que o próprio uso de RV, visto essa maior facilidade de operação por parte dos instaladores. De fato, o monitoramento do progresso da construção é um dos grandes benefícios da realidade aumentada e tem potencial para identificar possíveis falhas na instalação de detalhes de construção (DAVILA DELGADO et al., 2020).

\section{CONCLUSÕES}

A realidade aumentada é uma tecnologia que facilita na compreensão dos projetos dos sistemas hidrossanitários. O método de aplicação com o uso do Augin teve resultados satisfatórios, com precisão de $0,5 \mathrm{~cm}$. Os instaladores entrevistados pontuaram como excelente o uso dessa tecnologia visto que os modelos 3D são projetados diretamente no local da edificação em escala real 1:1. Além disso esses resultados foram satisfatórios em todos os padrões de qualidade - baixo, médio e alto - universalizando o uso na construção civil.

\section{REFERÊNCIAS}

ALTOQI. QiBuilder 2020 Basic Next Software. (2020). Disponível em: <https://altoqi.com.br/qibuilder/>.

AUGIN. Augin App. (2020). Disponível em: <https://augin.app/>. 
AZUMA, RONALD et al. Recent advances in augmented reality. Computer graphics and applications, IEEE, v. 21, n. 6, 2001.

BEHZADI, A. (2017). American Journal of Engineering Research ( AJER ) Open Access Using Augmented and Virtual Reality Technology in the Construction Industry Ajang Behzadi. American Journal of Engineering Research, 5(12), 350-353.

DALLASEGA, P., REVOLTI, A., SAUER, P. C., SCHULZE, F., \& RAUCH, E. (2020). BIM, augmented and virtual reality empowering lean construction management: $A$ project simulation game. Procedia Manufacturing, 45, 49-54.

DAVILA DELGADO, J. M., OYEDELE, L., BEACH, T., \& DEMIAN, P. (2020). Augmented and Virtual Reality in Construction: Drivers and Limitations for Industry Adoption. Journal of Construction Engineering and Management, 146(7), 04020079.

EL AMMARI, K., \& HAMMAD, A. (2019). Remote interactive collaboration in facilities management using BIM-based mixed reality. Automation in Construction, 107(August), 102940.

GARBETT, J., HARTLEY, T., \& HEESOM, D. (2021). A multi-user collaborative BIM-AR system to support design and construction. Automation in Construction, 122(November 2020), 103487.

MESQUITA, H. de C., EDUARDO, R. C., RODRIGUES, K. C., \& PAULA, H. M. de. (2018). Estudo de caso da análise de interferências entre as disciplinas de um edifício com projetos convencionais (re) modelados em BIM. Matéria (Rio de Janeiro), 23(3).

MOREIRA, L. C. de S. O Manual do Proprietário da Edificação Assistido pela Realidade Aumentada. 2018. 241 f. Tese (Doutorado em Arquitetura, Tecnologia e Cidade) - Faculdade de Engenharia Civil, Arquitetura e Urbanismo, Universidade Estadual de Campinas, Campinas, 2018.

SANTOS, A. S., SANTOS, F. E., CARVALHO, M. D. R., SILVA, W. R., SANTIN, K. R. S. (2020). Realidade aumentada na construção civil, aplicada para solucionar problemas e prever falhas de projetos e execução. 1-28.

SIDANI, A., DINIS, F. M., SANHUDO, L., DUARTE, J., SANTOS BAPTISTA, J., POÇAS MARTINS, J., \& SOEIRO, A. (2021). Recent Tools and Techniques of BIM-Based Virtual Reality: A Systematic Review. Archives of Computational Methods in Engineering, 28(2), 449-462.

SINDUSCON-MG. (2007). Custo Unitário básico (CUB/m2). 112. www.sinduscon-mg.org.br

RE, G. M., OLIVER, J., \& BORDEGONI, M. (2016). Impact of monitor-based augmented reality for onsite industrial manual operations. Cognition, Technology and Work, 18(2), 379-392.

YANG, D., DE VRIES, B., \& VAN DER SCHAFT, L. (2021). The construction workers' preference and acceptance of innovations in data provision: A stated choice experiment study in the Netherlands. Journal of Building Engineering, 35, 101970.

YEH, K.-C., TSAI, M.-H., \& KANG, S.-C. (2012). On-Site Building Information Retrieval by Using Projection-Based Augmented Reality. Journal of Computing in Civil Engineering, 26(3), 342-355. 